\title{
Prospective Prediction of Nonsuicidal Self-Injury: A I-Year Longitudinal Study in Young Adults
}

\author{
Catherine R. Glenn \\ Stony Brook University \\ E. David Klonsky \\ University of British Columbia
}

Nonsuicidal self-injury (NSSI) has become a significant public health problem. Although numerous studies have examined cross-sectional psychological correlates of NSSI, there has been little research examining predictors of NSSI over time. The present study examined cross-sectional and longitudinal correlates of NSSI in 81 young adult selfinjurers ( $M$ age $=19,74.1 \%$ female, $51.9 \%$ Caucasian), 51 of whom were followed up 1 year later. At baseline, participants completed self-report measures of NSSI, AxisI disorders, borderline personality disorder (BPD), and impulsivity, as well as an implicit measure of NSSI attitudes and identity. One year later, participants completed a Timeline Followback Method whereby they indicated their engagement in NSSI over the previous 12 months. Analyses replicated many known cross-sectional correlates of NSSI, including symptoms of several Axis-I disorders and BPD. However, many of these same variables failed to predict the course of NSSI over the 1-year follow-up. The only variables to prospectively predict NSSI were past NSSI (i.e., frequency, methods, and recency of NSSI), participants' behavioral forecast of their engagement in future NSSI, and BPD features. Findings suggest that many cross-sectional corre-

This study was supported in part by the Office of the Vice President for Research at Stony Brook University, and an NIH grant (MH08009601) awarded to E. David Klonsky, as well as a Psi Chi Graduate Research Grant and an NIH NRSA Predoctoral Fellowship Award to Catherine R. Glenn (F31-MH086197).

Address correspondence to E. David Klonsky, Ph.D., Department of Psychology, University of British Columbia, 2136 West Mall, Vancouver, BC, V6T 1Z4, Canada; e-mail: edklonsky@gmail.com 0005-7894/xx/xxx-xxx/\$1.00/0

() 2011 Association for Behavioral and Cognitive Therapies. Published by Elsevier Ltd. All rights reserved. lates of NSSI may not be useful for predicting subsequent NSSI. Instead, NSSI severity and BPD features appear to best predict continued engagement in NSSI.

Keywords: nonsuicidal self-injury; self-injurious behavior; deliberate self-harm; longitudinal

NONSUICIDAL SELF-INJURY (NSSI) REFERS to a class of behaviors defined by deliberate, direct, and selfinflicted tissue damage without suicidal intent and for purposes not socially sanctioned (e.g., skin cutting and burning; Favazza \& Conterio, 1989; International Society for the Study of Self-Injury, n.d.; Nock \& Prinstein, 2004; Whitlock, Eckenrode, \& Silverman, 2006). Lifetime rates of NSSI are particularly high among adolescents $(14-15 \%$ in high school samples; Laye-Gindhu \& SchonertReichl, 2005; Ross \& Heath, 2002) and young adults $(17 \%$ or more in college samples; Gratz, 2001; Whitlock et al., 2006). These high rates are alarming because NSSI is associated with many psychological disorders and may increase risk for suicide (Andover, Pepper, Ryabchenko, Orrico, \& Gibb, 2005; Favazza \& Conterio, 1989; Klonsky, Oltmanns, \& Turkheimer, 2003; Nock, Joiner, Gordon, Lloyd-Richardson, \& Prinstein, 2006).

Understanding the psychological correlates of NSSI is the first step toward identifying factors that cause and maintain the behavior. To date, a large body of literature has addressed cross-sectional correlates of NSSI. Replicated findings include associations between NSSI and depression, anxiety, eating disorders, substance-related disorders, and a 
variety of personality disorders, especially borderline personality disorder (BPD; Andover et al., 2005; Favazza \& Conterio, 1989; Klonsky et al., 2003). NSSI is also related to negative emotionality (Klonsky et al., 2003) and emotion dysregulation (Gratz \& Roemer, 2008), which are traits common to many clinical disorders, and therefore may explain NSSI's relationship to these diagnostic variables. In addition, although evidence linking NSSI and impulsivity is mixed, recent studies have found that NSSI is related to self-reported measures of impulsivity (Janis \& Nock, 2009), and specifically to certain impulsive traits, such as urgency (i.e., the tendency to commit rash action when faced with negative emotions) and lack of premeditation (i.e., the inability to delay action in order to deliberate and plan; Glenn \& Klonsky, 2010).

This growing body of data has led many to highlight the roles of known psychological correlates of NSSI, such as depression, anxiety, BPD, and impulsivity, in the maintenance and treatment of NSSI (Favazza, DeRosear, \& Conterio, 1989; Klonsky \& Muehlenkamp, 2007; Nixon, Adulakh, Townsend, \& Atherton, 2008; Nock, Temper, \& Hollander, 2007). Implicit is the assumption that cross-sectional correlates of NSSI are likely to play important roles in the course of NSSI over time, and in predicting who is likely to continue or stop engaging in NSSI. However, this assumption has not been addressed empirically. Almost all of the literature on NSSI correlates is cross-sectional, and the field lacks knowledge about which psychological variables predict continuation or cessation of NSSI over time.

There are good theoretical reasons why some of these cross-sectional correlates may be important for predicting a longitudinal course of NSSI. For example, insofar as emotion regulation is the main motivation for engaging in NSSI (Klonsky, 2007), it stands to reason that disorders characterized by intense emotional distress such as depression, anxiety, and BPD could help maintain the behavior over time. In addition, greater impulsive urgency, which reflects a tendency to act rashly when experiencing negative emotions, could indicate a vulnerability to continued engagement in maladaptive emotion regulation methods such as NSSI. Further, substance use (Wills \& Shiffman, 1985) and bulimia (Sim \& Zeman, 2005), two behavioral disorders used in part for emotion regulation purposes, could indicate increased propensity to engage in other maladaptive behaviors to regulate emotion such as NSSI, and thus an increased likelihood of continued NSSI.

Unfortunately, there has been little data examining these aforementioned variables as potential predictors of NSSI. To our knowledge only three studies have examined predictors of an NSSI course over time. ${ }^{1}$ Yates, Tracy, and Luthar (2008) demonstrated that, for female adolescents only, parental criticism predicted the onset of NSSI within the subsequent 6 years. Further analyses revealed that perceived parental alienation mediated this relationship. However, these variables did not prospectively predict the frequency of NSSI. A second study examined the role of temperament in predicting future NSSI in a sample of female patients with BPD enrolled in a clinical trial (Chapman, Derbidge, Cooney, Hong, \& Linehan, 2009). Findings suggest that, after controlling for prestudy NSSI, none of the temperament variables significantly predicted NSSI engagement over the subsequent 12-month period. Finally, in a treatment-seeking sample of adolescents, Guerry and Prinstein (2010) found that depressive symptoms were inversely related to NSSI remission in the 6 months following hospital discharge. Further, negative attributional style and stressful life events interacted to predict more NSSI between 9 and 18 months posttreatment.

Taken together, these three studies provide important, albeit preliminary, evidence regarding prospective predictors of NSSI. Interestingly, neither Yates et al. (2008) nor Guerry and Prinstein (2010) identified a robust predictor of future NSSI. Yates et al. found that perceived criticism predicted initiation of NSSI for girls but not boys. Guerry and Prinstein found that depression and the interaction between negative attributional style and stressful life events predicted NSSI during some follow-up intervals but not others. Only Chapman et al. (2009) identified a strong predictor, finding that past NSSI was the best predictor of future NSSI, a result consistent with the literature on prediction of future suicide attempts (Lewinsohn, Rohde, \& Seeley, 1994; Prinstein et al., 2008). Clearly, additional research on this topic is needed.

The current study sought to extend previous research on the prospective prediction of NSSI in a number of ways. First, this study examined the course of NSSI in a nonclinical sample, as compared

\footnotetext{
${ }^{1}$ Two additional studies that did not distinguish between nonsuicidal and suicidal self-injurious behaviors are not described here because it is not possible to determine whether their findings apply to NSSI, attempted suicide, or both (Brown, Comtois, Linehan, Murray, \& Chapman, 2009; Janis \& Nock, 2008). In addition, we did not include a longitudinal study conducted by Hilt, Nock, Lloyd-Richardson, and Prinstein (2008), which demonstrated that NSSI at Time 1 predicted increases in relationship quality with fathers at Time 2 , because it did not include measurement of NSSI at Time 2, and thus the course of NSSI, and predictors of NSSI course, could not be examined.
} 
to the treatment samples followed by Chapman et al. (2009) and Guerry and Prinstein (2010). Second, this study was conducted with young adult selfinjurers recruited from a university sample, which is a population that has not been studied in previous longitudinal research. Young adulthood is an important time period when both stopping and starting NSSI are common (Whitlock et al., 2006). Third, this study examined a range of known crosssectional correlates of NSSI that could potentially maintain the behavior over time. These include anxiety, depression, BPD, and impulsivity, as well as two other disorders that are linked with NSSI: bulimia (Favazza \& Conterio, 1989) and alcohol abuse (Favazza \& Conterio; Nock et al., 2006). In addition to diagnostic and personality variables, this study included a measure of implicit attitudes toward and self-identification with NSSI (i.e., SIIAT; Nock \& Banaji, 2007a). The SI-IAT has been shown to uniquely predict cross-sectional NSSI (Nock \& Banaji, 2007a) and to be one of the best longitudinal predictors of suicidal thoughts and behaviors (Nock et al., 2010; Nock \& Banaji, 2007b), although not NSSI specifically. Fourth, this study examined NSSI functions (in addition to NSSI behaviors) as prospective predictors of future NSSI. An examination of NSSI functions is important because previous research has demonstrated that particular NSSI functions (i.e., those providing intrapersonal reinforcement, such as affect regulation) are related to more severe NSSI and psychopathology (Klonsky \& Glenn, 2009; Nock \& Prinstein, 2005), and therefore may predict a more persistent course of NSSI.

Finally, this study considered the roles of past behavior and behavioral forecast as predictors of future NSSI. In the suicide literature, past suicidal behavior is one of the strongest predictors of future suicidal thoughts and behaviors (Lewinsohn et al., 1994; Prinstein et al., 2008). Chapman et al. (2009) provides evidence that the same may be true for NSSI. In addition, although Janis and Nock (2008) did not disambiguate nonsuicidal and suicidal selfinjurious thoughts and behaviors, their findings also suggest that past self-injury is a strong predictor of future self-injury. Further, Janis and Nock found that individuals' forecasts of their future self-injurious thoughts and behaviors predicted their future engagement in self-injurious thoughts and behaviors, but not over and above their past behavior. Therefore, the present study examined both the direct and unique effects of past NSSI and behavioral forecast of NSSI in predicting future NSSI. All of the variables included in this study were hypothesized to positively relate to NSSI engagement over the 1-year follow-up period.

\section{Method}

\section{PARTICIPANTS AND PROCEDURE}

\section{Baseline}

One thousand one hundred and ten college students from introductory psychology courses were screened for a history of 12 NSSI behaviors (e.g., skin cutting, burning) using a reliable and valid selfreport measure (see description of the Inventory of Statements About Self-Injury [ISAS] in the Measures section for psychometric information and a full list of NSSI behaviors assessed). Consistent with rates reported in previous studies of college populations (Klonsky \& Olino, 2008; Whitlock et al., 2006), 19.4\% of students reported having engaged in at least one NSSI behavior. These students were informed by e-mail about the opportunity to participate in a psychology experiment; this e-mail did not reveal that the study focused on NSSI. Of those contacted, $57.9 \%$ expressed interest in participating in a psychology study. These participants were then given additional information about the purpose and procedure of the study, including the study's focus on NSSI. From the interested pool of 125 potential self-injurers, 89 students $(71.2 \%)$ agreed to come into the lab for the study. Although most of the self-injurers who chose not to participate did not indicate a reason, six students who opted not to participate indicated that they felt uncomfortable talking about their self-injury.

The study protocol was approved by the University's Institutional Review Board and participant consent was obtained prior to the assessments at baseline and 1-year follow-up. The 89 self-injurers who were willing to participate completed the first part of the study (i.e., baseline) in a single lab visit. A brief structured interview (see Measures section) was used to confirm the history of NSSI reported on the self-report screening measure (i.e., ISAS). Based on this interview, eight participants were excluded from the self-injuring group because they did not meet the inclusion criteria for the study; these participants misunderstood the type of behaviors we were studying and, on the screening measure, included behaviors such as twirling hair, biting nails, or accidental injuries in their endorsement of NSSI. In addition to the sample of self-injurers, a noninjuring control sample of students, who had never engaged in an episode of NSSI, was also recruited from lowerlevel psychology courses. The final sample included 167 students: 81 confirmed self-injuring participants and 86 confirmed noninjuring control participants. At baseline, participants completed a battery of self-report questionnaires (i.e., ISAS, 
Patient Health Questionnaire [PHQ], McLean Screening Instrument for BPD [MSI-BPD], and UPPS Impulsive Behavior Scale [UPPS]), a brief structured interview about NSSI, and a behavioral measure of NSSI attitudes and identity (i.e., SI-IAT). At the end of the lab visit, the 81 self-injurers were asked whether they could be recontacted 1 year later; all 81 consented to be contacted again in 1 year.

\section{1-year Follow-Up}

One year later, self-injuring participants were recontacted to participate in the second, follow-up lab visit. Fifty-one of the original 81 self-injurers $(63 \%)$ participated in the follow-up $(M=380.6$ days, $S D=41.4)$, which is consistent with retention rates for longitudinal suicidality studies (i.e., $52-83 \%$; Boergers \& Spirito, 2003). Compared to the 30 selfinjurers who did not participate in the follow-up, the 51 self-injurers who did participate were not significantly different in age, gender, ethnicity, lifetime frequency of NSSI, NSSI functions, or whether they were engaging in NSSI at baseline (all ps ranged from .36 to .72). Reasons for participant attrition included: transfer to another university $(n=4)$, medical issue $(n=2)$, and explicitly stated that did not want to participate in follow-up $(n=1)$. In addition, five participants initially expressed interest in the follow-up study, but then never scheduled an appointment. The remaining 20 participants did not respond to any communication about the follow-up study. At follow-up, participants completed measures of NSSI over the past year (i.e., Timeline Followback Method for NSSI [TLFB-NSSI]).

\section{MEASURES}

\section{Inventory of Statements About Self-Injury}

The ISAS (Klonsky \& Glenn, 2009; Klonsky \& Olino, 2008) is a self-report measure that assesses the frequency and functions of NSSI. Recent research found the ISAS to be a reliable and valid measure of NSSI frequency and functions in a large sample of young adults (Klonsky \& Glenn; Klonsky \& Olino). The first section of the ISAS assesses the lifetime frequency of 12 different NSSI behaviors performed "intentionally (i.e., on purpose) and without suicidal intent," including banging/hitting, biting, burning, carving, cutting, interfering with wound healing, pinching, pulling hair, rubbing skin against rough surfaces, severe scratching, sticking self with needles, and swallowing dangerous chemicals. In addition, the questionnaire assesses some descriptive features of NSSI, including the age of NSSI onset and date of most recent NSSI. This section of the ISAS was used as the screening measure to recruit the self-injuring sample at baseline. The NSSI behaviors section of the ISAS has demonstrated good short-term (1-4 weeks) test-retest reliability $(r=.85)$, as well as construct validity indicated by theoretically consistent relationships to other NSSI and clinical variables (Klonsky \& Olino). Further, in previous studies, the 12 ISAS behaviors have demonstrated excellent internal consistency ( $\alpha=.84$; Klonsky \& Olino). In the current study the reliability estimate for the ISAS behaviors was lower $(n=51, \alpha=.58)$, likely due to the small sample size and the relatively low endorsement of some behaviors.

The second section of the ISAS measures the functions of NSSI. The ISAS assesses 13 functions of NSSI that have been proposed in the empirical and theoretical mental health literature (e.g., Chapman, Gratz, \& Brown, 2006; Klonsky, 2007). Consistent with previous research on NSSI functions (Nock \& Prinstein, 2004), the 13 ISAS functional subscales are well represented by a twofactor structure accounting for $61 \%$ of variance (Klonsky \& Glenn, 2009). The two superordinate factors are (a) intrapersonal functions (i.e., affect regulation, anti-dissociation, anti-suicide, marking distress, and self-punishment), and (b) interpersonal functions (i.e., autonomy, interpersonal boundaries, interpersonal influence, peer bonding, revenge, self-care, sensation seeking, and toughness; see Klonsky \& Glenn, 2009, for more information about factor structure). Each function is assessed based on participants' typical experience of NSSI (i.e., aggregating across all NSSI episodes and methods) with three items that are rated on a scale from 0 (not at all relevant) to 2 (very relevant) to the experience of NSSI. Therefore, each of the 13 functional subscale scores ranges from 0 to 6 . The two superordinate scales (i.e., intrapersonal and interpersonal) are derived by summing the subscales that belong to each superordinate scale (see above) and then dividing by the number of subscales in order to obtain a mean score. The functions section of the ISAS exhibits good construct validity (i.e., expected associations with theoretically related diagnostic variables and NSSI features; Klonsky \& Glenn, 2009). The ISAS functions demonstrated fair internal consistency in the follow-up sample ( $n=51$; intrapersonal $\alpha=.69$, interpersonal $\alpha=.75$ ).

\section{Brief Structured Interview for NSSI}

In addition to the ISAS, a brief structured interview for NSSI was designed for this study to (a) confirm a history of NSSI (i.e., NSSI behaviors were performed on purpose, not for suicidal reasons, and were severe in nature-bleeding, bruising, or caused 
pain), and (b) assess participants' behavioral forecast of their future NSSI (i.e., How likely is it that you will self-injure in the future? Rated on a scale from 1 [definitely NO] to 6 [definitely YES]).

\section{Patient Health Questionnaire}

Axis I clinical symptoms were assessed with the PHQ (Spitzer, Kroenke, \& Williams, 1999), an 83item self-report questionnaire that assesses the DSM-IV symptoms associated with four types of psychological problems: depression, anxiety, bulimia, and alcohol abuse. The PHQ has demonstrated excellent convergence with independent practitioner ratings (.85) and fair to excellent sensitivity (.75) and specificity (.90) in diagnosing Axis I disorders (Spitzer et al.). In the total sample, the PHQ scales demonstrated fair to good internal consistency: major depression $(\alpha=.85)$, generalized anxiety $(\alpha=.72)$, bulimia $(\alpha=.78)$, and alcohol abuse $(\alpha=.60)$.

\section{McLean Screening Instrument for BPD}

BPD symptoms were assessed using the MSI-BPD (Zanarini et al., 2003), a 10-item self-report measure of BPD features. Each DSM-IV BPD criterion is assessed with one item on the MSI$\mathrm{BPD}$, except for the paranoid ideation/dissociative symptoms criterion that is measured with two items on the MSI-BPD. The total MSI-BPD is a sum of 10 yes/no items. Compared to a validated structured interview, sensitivity and specificity of the MSI-BPD were excellent (both above .90) in a sample of young adults (Zanarini et al.). In an effort not to inflate the relationship between the MSI-BPD features and NSSI, we did not include the suicide/ self-injury item on the MSI-BPD in the main study analyses. In the total sample, the MSI-BPD items demonstrated excellent internal consistency with $(\alpha=.84)$ or without $(\alpha=.83)$ the suicide/self-injury item included.

\section{UPPS Impulsive Behavior Scale}

The UPPS (Whiteside \& Lynam, 2001) assesses four traits that lead to impulsive behavior: urgency, (lack of) perseverance, (lack of) premeditation, and sensation seeking. The full UPPS scale is composed of 45 items that are rated on a 4-point scale from 1 (agree strongly) to 4 (disagree strongly). This study utilized the UPPS 16-item short form that consists of four items from each impulsivity subscale. The short form was created by selecting the four items from each subscale that had the highest item-total correlations in the original study (Whiteside \& Lynam). Internal consistencies for the UPPS short form were very good in the present study ( $\alpha$ ranged from .79 to .85 for each of the four subscales). Intercorrelations among the subscales ranged from
-.01 to $.51(M d n=.16)$, suggesting that the subscales assess nonredundant aspects of impulsivity.

Self-Injury Implicit Association Test

The SI-IAT (Nock \& Banaji, 2007a) measures two types of implicit associations with NSSI: (a) attitudes toward NSSI (e.g., cutting: good or bad), and (b) identification with NSSI (e.g., cutting: me or not me). A previous study found that the SI-IAT significantly distinguished between adolescents who engaged in NSSI and those who did not (Nock \& Banaji). Specifically, adolescents who engaged in NSSI had a more accepting attitude toward NSSI and linked NSSI more closely with their self-image. In addition, the SI-IAT contributed unique information in predicting adolescents who engaged in NSSI over and above a range of demographic and psychiatric variables (Nock \& Banaji).

\section{Timeline Followback Method for NSSI}

The TLFB-NSSI was adapted from Sobell and Sobell (1992). In order to measure NSSI in the year between baseline and follow-up, we modified the Timeline Followback Method (TLFB), an interview that was designed by Sobell and Sobell (1992) for measuring alcohol consumption up to 24 months prior to the assessment. The original TLFB was designed to obtain quantitative retrospective reports of alcohol consumption using a calendar format (Sobell \& Sobell, 1992). The TLFB demonstrates good reliability and validity when used over a 1-year period, and has been endorsed by the American Psychiatric Association (Sobell \& Sobell, 2003). In addition, the TLFB has also been modified to investigate other clinically relevant behaviors, including problem gambling (Hodgins \& Makarchuk, 2003) and risky sexual behaviors (Weinhardt et al., 1998).

The current study adapted the original TLFB to assess NSSI behaviors over 1 year. At follow-up, self-injurers completed a TLFB for NSSI (TLFBNSSI) in questionnaire format, whereby they indicated their daily engagement in NSSI during the previous year (i.e., since their initial assessment at baseline). This calendar data was then utilized to compute NSSI frequency by summing the ranktransformed frequencies of each of the 12 NSSI behaviors performed between baseline and 1-year follow-up (rank-transformed rather than raw frequencies were utilized to minimize the influence of outliers). The NSSI behaviors assessed by the TLFB (excluding carving and swallowing dangerous chemicals that were not used by any participants over the follow-up year) demonstrated low internal consistency $(n=51, \alpha=.50)$, likely due to low 
endorsement of behaviors over the follow-up period.

\section{DATA ANALYTIC PLAN}

First, we assessed cross-sectional differences at baseline on demographic and clinical variables between self-injurers and noninjuring controls using independent samples $t$-tests and Pearson chisquare tests. Second, cross-sectional correlates of lifetime NSSI frequency were examined with Pearson correlations. The self-injuring sample size at baseline $(n=81)$ provided adequate power to detect significant correlations of $.31(\alpha=.05,1-\beta=.80)$ to $.37(\alpha=.01,1-\beta=.80)$ or higher. Third, we examined the relationship between baseline NSSI variables and NSSI frequency during the follow-up period using Pearson correlations. The self-injuring sample size at 1 -year follow-up $(n=51)$ provided adequate power to detect significant correlations of $.38(\alpha=.05,1-\beta=.80)$ to $.45(\alpha=.01,1-\beta=.80)$ or higher. Fourth, variables that significantly related to NSSI frequency over the 1-year follow-up were simultaneously entered into a linear regression. The observed power for the multiple regression analysis was adequate $(\alpha=.01,1-\beta=0.99$, number of predictors $=5, R^{2}=.48, n=51$ ). Finally, we utilized independent samples $t$-tests to examine variables that predicted remission among current injurers (i.e., NSSI within 6 months prior to baseline), and relapse among past injurers (i.e., no NSSI within 6 months prior to baseline). Due to the large number of planned analyses conducted, we were mindful of the risk of Type I error. We therefore present both effect sizes and $p$ values for all analyses so that results can be interpreted in terms of both magnitude and statistical reliability/significance. We utilized this approach throughout the Results section.

\section{Results}

DESCRIPTIVE CHARACTERISTICS OF NSSI AMONG SELF-INJURERS AT BASELINE

For self-injuring participants assessed at baseline, the average age of NSSI onset was 13 years old $(S D=2.9)$. The most common NSSI behaviors were cutting (performed by $82.7 \%$ of sample) and banging/hitting ( $70.4 \%$ of sample). However, almost all self-injurers $(96 \%)$ reported engaging in more than one NSSI method over their lifetime and most engaged in three or more NSSI methods $(81 \%)$. The NSSI behaviors with the highest frequency were pulling hair ( $M$ total sample $=80.5$, $S D=555.3)$, cutting $(M$ total sample $=62.5$, $S D=170.3)$, and severe scratching ( $M$ total sample $=44.8, S D=231.1)$. In regard to recency of NSSI, $52 \%$ of self-injurers had engaged in NSSI in the 6 months prior to baseline assessment (which was used as the cutoff for assigning "current" NSSI status at baseline), and $27.8 \%$ had engaged in NSSI during the month prior to the baseline assessment.

\section{CROSS-SECTIONAL CORRELATES OF NSSI STATUS AT BASELINE}

Table 1 displays the demographic and clinical characteristics of the self-injuring and noninjuring control group at baseline. The demographic, diagnostic (PHQ), and impulsivity (UPPS) differences between the self-injuring group and noninjuring control group at baseline were previously published in Glenn \& Klonsky, 2010. We summarize these findings briefly here because they provide an important context for understanding the focus of this article, which is the utility of baseline variables for prospectively predicting NSSI. In addition, we present new data (i.e., BPD features and IAT results) that have not been previously published.

The self-injuring group was somewhat younger $(p=.014)$ than the control group. As for diagnostic variables, the self-injuring group had significantly more participants who met the DSM-IV criteria threshold (as indexed by self-reported symptoms on the PHQ) for alcohol abuse $(p=.003)$, generalized anxiety $(p=.003)$, and major depression $(p=.022)$. In addition, the self-injuring group endorsed significantly more BPD features on the MSI-BPD than controls (even if the suicide/self-injury item was omitted; $p s<.001$ ). In regard to impulsivity, self-injurers endorsed greater impulsive urgency (i.e., the tendency to act rashly in the face of negative affect; $p=.001$ ), lack of premeditation (i.e., the inability to delay action in order to deliberate and plan; $p=.007$ ), and sensation seeking (i.e., the tendency to seek excitement; $p=.026$ ) on the UPPS compared to the noninjuring control group.

In order to examine implicit associations with NSSI, we examined differences between self-injurers and controls on the SI-IAT. On the identity version of the SI-IAT (i.e., cutting associated with me or not me), the self-injuring group identified themselves significantly more with NSSI than the control group $(p=.003)$. Further, on the attitude version of the SI-IAT (i.e., cutting associated with good or bad), there was a trend for the self-injuring group to indicate that NSSI was less unfavorable than the control group $(p=.070)$, but this difference did not reach statistical significance.

\section{CROSS-SECTIONAL CORRELATES OF NSSI FREQUENCY AT BASELINE}

Correlates of lifetime NSSI frequency are presented in Table 2. Lifetime NSSI frequency was created by rank-transforming frequencies of each of the 12 
PROSPECTIVE PREDICTION OF NSSI

Table 1

Demographic and Clinical Characteristics of Self-Injurers and Controls at Baseline

\begin{tabular}{|c|c|c|c|c|c|}
\hline Variable $^{a}$ & $\begin{array}{l}\text { NSSI } \\
(n=81)\end{array}$ & $\begin{array}{l}\text { Control } \\
(n=86)\end{array}$ & Statistical Test ${ }^{\mathrm{b}}$ & $p$ & Effect Size \\
\hline \multicolumn{6}{|l|}{ Demographics } \\
\hline Age: $M(S D)$ & $19.06(1.89)$ & $19.84(2.11)$ & $t=2.50$ & .014 & $d=0.39$ \\
\hline Gender: \% female & $74.1 \%$ & $69.8 \%$ & $x^{2}=0.20$ & .655 & $\Phi=.05$ \\
\hline Ethnicity: \% Caucasian & $51.9 \%$ & $37.2 \%$ & $x^{2}=3.06$ & .080 & $\Phi=.15$ \\
\hline \multicolumn{6}{|c|}{ Axis I Psychopathology (\# of participants who met full DSM-IV symptoms of disorder on PHQ) } \\
\hline Alcohol abuse & 28 & 12 & $x^{2}=8.63$ & .003 & $\Phi=.24$ \\
\hline Bulimia & 5 & 1 & $x^{2}=1.75$ & .186 & $\Phi=.13$ \\
\hline Generalized anxiety & 12 & 1 & $x^{2}=9.01$ & .003 & $\Phi=.25$ \\
\hline Major depression & 15 & 5 & $x^{2}=5.24$ & .022 & $\Phi=.20$ \\
\hline \multicolumn{6}{|c|}{ Personality and Impulsivity Variables } \\
\hline MSI-BPD features: $M(S D)$ & $6.31(2.36)$ & $2.07(2.26)$ & $t=11.75$ & $<.001$ & $d=1.83$ \\
\hline (w/out self-injury item) & $5.31(2.36)$ & $2.07(2.26)$ & $t=8.98$ & $<.001$ & $d=1.40$ \\
\hline \multicolumn{6}{|l|}{ Impulsivity: $M(S D)$} \\
\hline Urgency & $11.04(3.10)$ & $9.45(2.98)$ & $t=3.37$ & .001 & $d=0.52$ \\
\hline (lack of) Perseverance & $7.49(2.84)$ & $7.20(2.45)$ & $t=0.72$ & .471 & $d=0.11$ \\
\hline (lack of) Premeditation & $8.44(7.31)$ & $7.31(2.52)$ & $t=2.72$ & .007 & $d=0.42$ \\
\hline Sensation seeking & $11.51(3.53)$ & $10.30(3.38)$ & $t=2.25$ & .026 & $d=0.35$ \\
\hline \multicolumn{6}{|l|}{ Implicit Associations (SI-IAT) } \\
\hline Identity version & $-.09(.31)$ & $-.23(.29)$ & $t=3.07$ & .003 & $d=0.48$ \\
\hline Attitude version & $-.33(.28)$ & $-.42(.31)$ & $t=1.82$ & .070 & $d=0.28$ \\
\hline
\end{tabular}

a Detailed demographic, diagnostic (PHQ), and impulsivity (UPPS) differences between the self-injuring group and noninjuring control group at baseline were previously published in Glenn and Klonsky (2010).

b Independent samples $t$-test degrees of freedom $=165$. Pearson chi-square test degrees of freedom $=1, N=167$.

NSSI behaviors assessed by the ISAS and summing them to create a total frequency score. Rank transformations were used to reduce the influence of outliers. Regarding NSSI characteristics, lifetime NSSI frequency was positively correlated with the total number of NSSI methods used (e.g., cutting, burning), the total duration of NSSI (i.e., total number of years an individual has/had engaged in NSSI), behavioral forecast of future NSSI (i.e., participants' prediction of how likely it was that they would engage in NSSI in that next year), and the number of intrapersonal NSSI functions endorsed (e.g., affect regulation; all $p s<.001$ ). However, lifetime NSSI frequency was not significantly related to how many interpersonal functions NSSI served (e.g., peer bonding) nor to how recently an individual had engaged in NSSI ( $p=.457$ and .059 , respectively). In addition, the SI-IAT identity and attitude versions were not related to lifetime NSSI frequency $(p=.390$ and .995 , respectively).

Next, we examined the relationship between lifetime NSSI frequency and a range of diagnostic and personality variables known to be associated with NSSI history. As can be seen in Table 2, lifetime NSSI frequency was positively associated with BPD features $(p<.001)$, bulimia symptoms $(p=.005)$, and to a lesser degree with PHQ depressive symptoms and the lack of perseverance facet of impulsivity ( $p=.036$ and .018 , respectively). However, NSSI frequency was not significantly related to anxiety symptoms, alcohol abuse symptoms, nor to the three remaining UPPS impulsivity scales ( $p$ s ranged from .075 to .826).

\section{LONGITUDINAL SAMPLE: DESCRIPTIVE CHARACTERISTICS OF NSSI}

Longitudinal analyses focused on the 51 self-injurers who completed the 1-year follow-up assessment. As indicated in the Methods section, these 51 participants did not significantly differ from the 30 who did not participate in the follow-up on any demographic or baseline NSSI variables. The 51 self-injurers who participated at the 1-year follow-up had an average age of 18.96 years $(S D=1.57)$ and were $72.5 \%$ female. The ethnic make-up of this sample was 54.9\% Caucasian, 21.6\% Asian, 7.8\% Hispanic, $5.9 \%$ African American, and $9.8 \%$ "other" or mixed ethnic background.

During the 1-year follow-up period, 32 of the 51 participants $(62.7 \%)$ engaged in at least one NSSI behavior, and half of these participants engaged in two or more NSSI methods. For the 32 who engaged in NSSI during the follow-up period, the most common behaviors were banging/hitting self (performed by $46.9 \%$ ) and cutting $(43.7 \%)$, 
Table 2

Cross-Sectional and Longitudinal Correlates of NSSI

\begin{tabular}{|c|c|c|}
\hline Baseline Predictors & $\begin{array}{l}\text { Lifetime NSSI Frequency } \\
\text { at Baseline }{ }^{\text {a }}(N=81)\end{array}$ & $\begin{array}{l}\text { NSSI Frequency Between } \\
\text { Baseline and } 1 \text {-Year } \\
\text { Follow-Up }{ }^{b}(N=51)\end{array}$ \\
\hline \multicolumn{3}{|l|}{ NSSI Variables } \\
\hline Lifetime NSSI frequency & & $.45^{\star \star \star}$ \\
\hline Lifetime NSSI methods & $.93^{\star \star \star}$ & $.54^{\star * \star}$ \\
\hline Total years engaged in NSSI & $.44^{\star \star \star}$ & .16 \\
\hline Recency of $\mathrm{NSSI}^{\mathrm{C}}$ & -.21 & $-.38^{* *}$ \\
\hline Behavioral forecast of future NSSI & $.40^{\star \star \star}$ & $.37^{\star \star}$ \\
\hline NSSI intrapersonal functions & $.43^{\star \star \star}$ & .26 \\
\hline NSSI interpersonal functions & .08 & .05 \\
\hline SI-IAT identity version & .10 & -.13 \\
\hline SI-IAT attitude version & -.01 & .01 \\
\hline \multicolumn{3}{|l|}{ Diagnostic and Impulsivity Variables } \\
\hline PHQ alcohol abuse dimensional & .09 & -.01 \\
\hline PHQ bulimia dimensional & $.31^{\star *}$ & .27 \\
\hline PHQ generalized anxiety dimensional & .03 & .16 \\
\hline PHQ major depression dimensional & $.23^{*}$ & .16 \\
\hline MSI-BPD features ${ }^{d}$ & $.39^{\star * *}$ & $.41^{\star *}$ \\
\hline UPPS urgency & .07 & .08 \\
\hline UPPS (lack of) perseverance & $.26^{*}$ & .11 \\
\hline UPPS (lack of) premeditation & .20 & .25 \\
\hline UPPS sensation seeking & .17 & .01 \\
\hline \multicolumn{3}{|c|}{$\begin{array}{l}\text { a Lifetime frequency of NSSI behaviors was created by rank transforming each of the } 12 \text { NSSI behaviors (so that each behavior had the } \\
\text { same mean) and then summing the } 12 \text { rank-transformed behavior scores to obtain a total frequency score. } \\
\text { b Follow-up NSSI frequency (between baseline and 1-year follow-up) was created by rank transforming each of the } 12 \text { NSSI behaviors (so }\end{array}$} \\
\hline \multirow{3}{*}{\multicolumn{3}{|c|}{$\begin{array}{l}\text { that each behavior had the same mean) and then summing the } 12 \text { rank-transformed behavior scores to obtain a total frequency score. } \\
{ }^{c} \text { Recency of NSSI indicates how many months it had been since an individual engaged in NSSI. } \\
{ }^{d} \text { MSI-BPD features total score did not include the suicide/self-injury item. } \\
{ }_{*}^{*} p<.05 \text {. }\end{array}$}} \\
\hline & & \\
\hline & & \\
\hline \multicolumn{3}{|l|}{${ }^{* *} p<.01$} \\
\hline$\star * \star \quad p<.001$ & & \\
\hline
\end{tabular}

followed by pulling hair $(25 \%)$, severe scratching $(21.9 \%)$, burning $(18.7 \%)$, interfering with wound healing $(15.6 \%)$, and biting $(12.5 \%)$. The following behaviors were only performed by one participant over the follow-up period: pinching, rubbing skin against rough surfaces, and sticking self with needles. No participants engaged in carving or swallowing dangerous chemicals over the follow-up. The NSSI behaviors with the highest frequency were biting $(M=11.5, S D=64)$, banging/hitting $(M=8.8$, $S D=27.4)$, pulling hair $(M=8.7, S D=40.2)$, and cutting $(M=5.4, S D=12)$.

Of the 51 self-injurers assessed at Time 2, 15 $(29.4 \%)$ reported seeing a mental health professional at some point between Time 1 and Time 2 (similar to rates of treatment seeking in community samples for disorders such as depression [36\%] and anxiety [26\%]; Epidemiologic Catchment Area Study; Robins \& Regier, 1991). NSSI frequency during the follow-up did not differ between the selfinjurers who sought treatment $(M=26.80$,
$S D=37.70)$ compared to self-injurers who did not seek treatment $(M=25.08, S D=71.3), t(49)=0.09$, $p=.930, d=0.03$.

\section{LONGITUDINAL SAMPLE: PROSPECTIVE PREDICTION OF NSSI}

Next, we examined the cross-sectional correlates from Table 2 (column 1) as potential prospective predictors of NSSI. NSSI frequency between baseline and 1-year follow-up was utilized as the dimensional outcome measure. Consistent with the procedure for lifetime NSSI frequency, NSSI frequency in the follow-up year was computed by summing the rank-transformed frequencies for each of the 12 NSSI behaviors. Again, we utilized ranktransformed rather than raw frequencies in computing follow-up NSSI frequency to minimize the influence of outliers. A series of Pearson correlations were conducted between all cross-sectional correlates and NSSI frequency during the follow-up period. 
Of the variables that significantly distinguished self-injurers from controls (see Cross-sectional Correlates of NSSI Status at Baseline section), and that significantly related to frequency of NSSI at baseline (see Table 2, column 1), only five baseline variables were significantly related to NSSI frequency in the follow-up year: lifetime NSSI frequency $(p=.001)$, lifetime NSSI methods $(p<.001)$, recency of NSSI $(p=.007)$, behavioral forecast of future NSSI $(p=.006)$, and BPD features $(p=.003)$. In contrast, the remaining 13 NSSI and diagnostic cross-sectional correlates did not relate to NSSI frequency during the follow-up (all ps>.06).

Next, we examined the unique contributions of the significant predictors. The five variables that significantly predicted NSSI frequency over the 1-year follow-up were simultaneously entered into a linear regression (see Table 3). Together the five variables accounted for $48 \%$ of the variance in follow-up NSSI frequency. However, only lifetime NSSI methods $(p=.006)$, BPD features $(p=.005)$, and, to a lesser degree, lifetime NSSI frequency $(p=.046)$, accounted for unique variance in followup NSSI frequency.

Finally, we explored the longitudinal course of NSSI in terms of categorical outcomes of relapse and remission based on NSSI status at baseline (i.e., current-NSSI within 6 months prior to baseline vs. past-no NSSI within 6 months prior to baseline). Although this approach has the disadvantage of losing variability captured by dimensional outcome variables, predicting relapse and remission can be useful clinically, and we therefore include these analyses as a supplement to the results reported above. First, to examine predictors of remission, current self-injurers at baseline (i.e., NSSI in
6 months prior to baseline) who continued to engage in NSSI during the 1-year follow-up (NSSI continuers, $n=23$ ) were compared to current selfinjurers at baseline who did not engage in NSSI during the 1-year follow-up (NSSI remitters, $n=5)$. NSSI continuers were distinguished from NSSI remitters by more lifetime NSSI methods, $M(S D)=5.61(1.85)$ versus $M(S D)=2.60(1.14)$; $t(26)=3.46, p=.002, d=1.36$, and greater lifetime NSSI frequency, $M(S D)=545.52(102.62)$ versus $M \quad(S D)=422.20 \quad(49.21) ; t(26)=2.59, p=.015$, $d=1.02$. In addition, current baseline females $(n=21)$ were more likely to continue to engage in NSSI during the follow-up period than current baseline males, $n=7 ; \chi^{2}(1, N=28)=3.98, p=.046, \Phi=.40$. There were no other significant differences between the NSSI continuers and NSSI remitters on the remaining demographic, NSSI, diagnostic, or impulsivity variables ( $p s$ ranged from .072 to .910).

Second, to examine predictors of relapse, past self-injurers at baseline (i.e., no NSSI in 6 months prior to baseline) who abstained from NSSI during the 1-year follow-up (NSSI abstainers, $n=14$ ) were compared to the past self-injurers at baseline who engaged in NSSI in the follow-up period (NSSI relapsers, $n=9$ ). Analyses revealed that prior to baseline, NSSI abstainers had been free of NSSI for significantly longer than NSSI relapsers, $M(S D)=$ 27.86 (15.59) months prior to baseline versus $M \quad(S D)=13.63$ (6.84) months; $t(26)=2.95$, $p=.008, d=1.16$. That is, self-injurers who continued to abstain from NSSI during the 1-year followup period had not self-injured on average for over 2 years, whereas self-injurers who relapsed during the follow-up period had stopped self-injuring an average of 1 year prior to baseline. NSSI abstainers and relapsers were not significantly different on any

Table 3

Unique Prediction of NSSI Frequency Over the 1-Year Follow-Up

\begin{tabular}{|c|c|c|c|}
\hline \multirow[b]{2}{*}{ Baseline Predictors } & \multicolumn{3}{|c|}{$\begin{array}{l}\text { NSSI Frequency Between Baseline and } \\
\text { 1-Year Follow-Up }{ }^{\text {a }}\end{array}$} \\
\hline & $\beta$ & $t$ & $p$ \\
\hline Lifetime NSSI frequency ${ }^{b}$ & -.65 & -2.06 & .046 \\
\hline Lifetime NSSI methods & .86 & 2.91 & .006 \\
\hline Recency of NSSI & -.21 & -1.69 & .098 \\
\hline Behavioral forecast of future NSSI & .19 & 1.39 & .171 \\
\hline MSI-BPD features ${ }^{c}$ & .37 & 2.96 & .005 \\
\hline All Variables Entered Simultaneously & \multicolumn{3}{|c|}{$R^{2}=.48, F(5,45)=7.94, p<.001$, Cohen's $f^{2}=0.92$} \\
\hline
\end{tabular}

\footnotetext{
${ }^{a}$ Follow-up NSSI frequency (between baseline and 1-year follow-up) was created by rank transforming each of the 12 NSSI behaviors (so that each behavior had the same mean) and then summing the 12 rank-transformed behavior scores to obtain a total frequency score.

b Lifetime frequency of NSSI behaviors was created by rank transforming each of the 12 NSSI behaviors (so that each behavior had the same mean) and then summing the 12 rank-transformed behavior scores to obtain a total frequency score.

${ }^{c}$ MSI-BPD features total score did not include the suicide/self-injury item.
} 
of the other demographic, NSSI, diagnostic, or impulsivity variables ( $p$ s ranged from .061 to .832).

\section{Discussion}

Numerous psychological correlates of NSSI have been identified via cross-sectional research. However, the vast majority of these variables have not been examined as predictors of NSSI in prospective designs. The current study examined correlates of NSSI both cross-sectionally and longitudinally over a 1-year period. Cross-sectional analyses largely replicated previous findings indicating that selfinjurers are characterized by elevated depression, anxiety, alcohol abuse, BPD features, and impulsivity (Andover et al., 2005; Favazza \& Conterio, 1989; Janis \& Nock, 2009; Klonsky et al., 2003; Nock et al., 2006). In addition, findings from the SIIAT mainly replicate those of Nock and Banaji (2007a), suggesting that self-injurers associate NSSI more with their identity. Also consistent with previous research (Klonsky \& Glenn, 2009; Nock \& Prinstein, 2005), lifetime frequency of NSSI was related to greater endorsement of intrapersonal functions (e.g., emotion regulation), more severe clinical symptoms (e.g., BPD features, bulimia, and depression to some degree), and, to a lesser extent, low perseverance (i.e., the inability to stay with a task through completion).

However, a different pattern of results emerged in the longitudinal portion of the study. A significant finding from the current study was that the majority of variables that distinguished self-injurers from controls cross-sectionally did not predict the course of NSSI over time. Specifically, depression, anxiety, bulimia, alcohol abuse, impulsivity, implicit associations with NSSI, and the functions of NSSI all were nonsignificant predictors of NSSI course despite relating to cross-sectional NSSI status in this and several previous studies (Andover et al., 2005; Nock et al., 2006; Nock \& Banaji, 2007a). The only variables that did significantly predict NSSI course were (a) past NSSI (specifically, lifetime NSSI frequency, lifetime NSSI methods, and recency of last NSSI episode), (b) an individual's forecast of his or her future NSSI behavior, and (c) BPD features. Of these, lifetime NSSI methods and BPD features were the only variables to uniquely predict subsequent NSSI.

Our finding that past NSSI predicts future NSSI is consistent with previous studies on prospective predictors of self-injurious behavior (Chapman et al., 2009; Janis \& Nock, 2008; Lewinsohn et al., 1994; Prinstein et al., 2008). Our results also extend this literature by demonstrating that the pattern generalizes to nonclinical self-injuring populations, and by suggesting that the number of NSSI methods may be the most important aspect of past NSSI (as compared to frequency or recency) for predicting subsequent NSSI. This is consistent with previous cross-sectional research demonstrating that the number of NSSI methods predicts psychopathology better than NSSI frequency (Nock et al., 2006).

Interestingly, although an individual's forecast of his or her future NSSI was significantly related to subsequent frequency of NSSI, it did not provide information over and above other significant predictors. Taken together with Janis and Nock (2008), our findings help clarify the utility of behavioral forecasts for predicting future NSSI. In both studies, behavioral forecast predicted future self-injurious behaviors but did not provide incremental information over and above past self-injury. In a clinical context, these findings suggest that behavioral forecasts of self-injurious behaviors may have limited utility for predicting likelihood of remission once past history of NSSI has been obtained.

The potential predictive ability of BPD features is also noteworthy. Self-reported BPD features prospectively predicted NSSI even though the suicide/ self-injury criterion was omitted from the BPD measure. Although previous work has shown strong cross-sectional relationships between NSSI and BPD (Andover et al., 2005; Klonsky et al., 2003), to our knowledge this is the first study to document that BPD features significantly predict the course of NSSI among those who self-injure. Moreover, even though not all self-injurers have a BPD diagnosis, these findings suggest that BPD symptoms may be a poor prognostic indicator of NSSI course and thus indicate a need for more aggressive monitoring and treatment of NSSI. However, given that BPD features were assessed using a validated self-report measure, future studies should seek to replicate these results using a semistructured interview to assess BPD symptoms.

It is surprising that the SI-IAT did not prospectively predict NSSI in this sample given that it has distinguished self-injurers from controls (Nock \& Banaji, 2007a) and uniquely predicted suicidal behavior (Nock et al., 2010; Nock \& Banaji, 2007b). It is possible that the nature of the current sample (i.e., nonclinical, young adults compared to nonclinical adolescents in Nock \& Banaji, 2007b, or clinical adults in Nock et al., 2010) could have contributed to these discrepant results. It is also possible that the SI-IAT predicts NSSI less robustly than suicidal behavior. In contrast to the prediction of suicidal behavior, only one cross-sectional study (Nock \& Banaji, 2007a) and no prospective studies have examined the SI-IAT in relation to NSSI. Given that our study failed to replicate some of the 
findings from Nock and Banaji (2007a), future research should continue to examine the value of the SI-IAT for predicting NSSI, including predictive utility differences across self-injuring groups (e.g., younger vs. older, nonclinical vs. clinical), as well as its usefulness as a prospective predictor of NSSI.

Finally, although the exploratory analyses predicting NSSI relapse and remission are preliminary and relied on small subsamples, these findings provide information that may be useful clinically as well as for informing future research. Among current self-injurers, remission 1 year later was most strongly predicted by a less severe history of NSSI (i.e., fewer NSSI methods and less lifetime NSSI frequency). Among past self-injurers, those who avoided relapse had on average stopped selfinjuring over 2 years prior to the beginning of the study, whereas those who relapsed during the follow-up had on average stopped self-injuring 1 year prior to the beginning of the study. Although the small sample permits only tentative inferences, it is possible that 1 year of NSSI abstinence does not yet signal full recovery, whereas 2 years of abstinence is a better indicator of genuine NSSI remission. Future research should examine periods of abstinence that predict continued recovery from NSSI.

This study represents an important contribution to the literature because it is the first to examine a wide range of psychological predictors in relation to the natural course of NSSI. However, several important limitations deserve comment. First, the self-injurers were young adults from a college sample. The sample's average age (19) is a strength because many self-injurers start self-injuring or stop self-injuring around this age (Whitlock et al., 2006). However, use of a college sample requires that additional research seek to replicate findings in more severe, younger, and other nonclinical selfinjuring groups. Second, clinical variables were assessed with validated self-report measures. It would be helpful to utilize structured and semistructured interview measures of psychopathology in future studies since these "gold standards" in diagnostic measurement offer improved validity. Third, certain aspects of the study design increased the risk of Type I and Type II error. The possibility of Type I error was increased due to the number of analyses conducted. In addition, the sample size was small for some analyses and therefore statistical power to detect effects was less than desirable. Further, the measures of NSSI behaviors (i.e., ISAS and TLFB) demonstrated lower reliability in the current sample than in previous studies (e.g., Klonsky \& Olino, 2008). Lower reliability is likely due in part to a small sample size and in part to relatively low endorsement of some NSSI behaviors. As a result, it is possible that the magnitude of some effect sizes was underestimated. Fourth, this study focused on variables likely to predict continued NSSI rather than protect against it. Future studies should also examine protective factors that may lead to a decline in NSSI, such as social support or positive coping strategies.

Finally, the five statistically significant prospective predictors accounted for $48 \%$ of the variance in NSSI, leaving a substantial portion of the variance unexplained. These results are consistent with previous research; for example, Chapman et al.'s (2009) prediction model accounted for $45 \%$ of the variance in NSSI. Future studies should examine additional variables that may improve prediction of NSSI course. For example, self-derogation/selfcriticism is a strong cross-sectional correlate of NSSI (Glassman, Weierich, Hooley, Deliberto, \& Nock, 2007; Klonsky et al., 2003), relevant to the primary functions of NSSI (Klonsky, 2007; Klonsky \& Glenn, 2009), and therefore may be an important predictor of NSSI course.

\section{References}

Andover, M. S., Pepper, C. M., Ryabchenko, K. A., Orrico, E. G., \& Gibb, B. E. (2005). Self-mutilation and symptoms of depression, anxiety, and borderline personality disorder. Suicide and Life-Threatening Behavior, 35, 581-591.

Boergers, J., \& Spirito, A. (2003). Follow-up studies of child and adolescent suicide attempters. In R. A. King \& A. Apter (Eds.). Suicide in children and adolescents (pp. 271-293). New York: Cambridge University Press.

Brown, M. Z., Comtois, K. A., Linehan, M. M., Murray, A., \& Chapman, A. L. (2009). Shame as prospective predictors of self-inflicted injury in borderline personality disorder: A multi-modal analysis. Behaviour Research and Therapy, 47, 815-822.

Chapman, A. L., Derbidge, C., Cooney, E., Hong, P., \& Linehan, M. M. (2009). Temperament as a prospective predictor of self-injury among patients with borderline personality disorder. Journal of Personality Disorders, 23, 122-140.

Chapman, A. L., Gratz, K. L., \& Brown, M. Z. (2006). Solving the puzzle of deliberate self-harm: The experiential avoidance model. Behaviour Research and Therapy, 44, 371-394.

Favazza, A. R., \& Conterio, K. (1989). Female habitual selfmutilators. Acta Psychiatrica Scandinavica, 79, 282-289.

Favazza, A. R., DeRosear, L., \& Conterio, K. (1989). Self-mutilation and eating disorders. Suicide and LifeThreatening Behavior, 19, 352-361.

Glassman, L. H., Weierich, M. R., Hooley, J. M., Deliberto, T. L., \& Nock, M. K. (2007). Child maltreatment, nonsuicidal self-injury, and the mediating role of self-criticism. Behaviour Research and Therapy, 45, 2483-2490.

Glenn, C. R., \& Klonsky, E. D. (2010). A multimethod analysis of impulsivity in non-suicidal self-injury. Personality Disorders: Theory, Research, and Treatment, 1, 67-75.

Gratz, K. L. (2001). Measurement of deliberate self-harm: Preliminary data on the Deliberate Self-Harm Inventory. Journal of Psychopathology and Behavioral Assessment, 23, 253-263. 
Gratz, K. L., \& Roemer, L. (2008). The relationship between emotion dysregulation and deliberate self-harm among female undergraduate students at an urban commuter university. Cognitive and Behaviour Therapy, 37, 14-25.

Guerry, J. D., \& Prinstein, M. J. (2010). Longitudinal prediction of adolescent non-suicidal self-injury: Examination of a cognitive vulnerability-stress model. Journal of Clinical Child and Adolescent Psychology, 39, 1-13.

Hilt, L. M., Nock, M. K., Lloyd-Richardson, E. E., \& Prinstein, M. J. (2008). Longitudinal study of non-suicidal self-injury among young adolescents. Journal of Early Adolescence, 28, 455-469.

Hodgins, D. C., \& Makarchuk, K. (2003). Trusting problem gamblers: Reliability and validity of self-reported gambling behavior. Psychology of Addictive Behaviors, 17, 244-248.

International Society for the Study of Self-Injury. (n.d.). About self-injury. Retrieved from http://www.isssweb.org/ aboutnssi.php

Janis, I. B., \& Nock, M. K. (2008). Behavioral forecasts do not improve the prediction of future behavior: A prospective study of self-injury. Journal of Clinical Psychology, 64, 1164-1174.

Janis, I. B., \& Nock, M. K. (2009). Are self-injurers impulsive? Results from two behavioral laboratory studies. Psychiatry Research, 169, 261-267.

Klonsky, E. D. (2007). The functions of deliberate self-injury: A review of the evidence. Clinical Psychology Review, 27, 226-239.

Klonsky, E. D., \& Glenn, C. R. (2009). Assessing the functions of non-suicidal self-injury: Psychometric properties of the Inventory of Statements About Self-Injury (ISAS). Journal of Psychopathology and Behavioral Assessment, 31, 215-219.

Klonsky, E. D., \& Muehlenkamp, J. J. (2007). Self-injury: A research review for the practitioner. Journal of Clinical Psychology, 63, 1045-1056.

Klonsky, E. D., \& Olino, T. M. (2008). Identifying clinically distinct subgroups of self-injurers among young adults: A latent class analysis. Journal of Consulting and Clinical Psychology, 76, 22-27.

Klonsky, E. D., Oltmanns, T. F., \& Turkheimer, E. (2003). Deliberate self-harm in a nonclinical population: Prevalence and psychological correlates. American Journal of Psychiatry, 160, 1501-1508.

Laye-Gindhu, A., \& Schonert-Reichl, K. A. (2005). Nonsuicidal self-harm among community adolescents: Understanding the "whats" and "whys" of self-harm. Journal of Youth and Adolescence, 34, 447-457.

Lewinsohn, P. M., Rohde, P., \& Seeley, J. R. (1994). Psychosocial risk factors for future adolescent suicide attempts. Journal of Consulting and Clinical Psychology, 62, 297-305.

Nixon, M. K., Adulakh, H., Townsend, L., \& Atherton, M. (2008). Psychosocial interventions for adolescents. In M. K. Nixon \& N. Heath (Eds.). Self-injury in youth: The essential guide to assessment and intervention (pp. 217-236). New York: Routledge.

Nock, M. K., \& Banaji, M. R. (2007). Assessment of selfinjurious thoughts using a behavioral test. American Journal of Psychiatry, 164, 820-823.

Nock, M. K., \& Banaji, M. R. (2007). Prediction of suicidal ideation and attempts among adolescents using a brief performance-based test. Journal of Consulting and Clinical Psychology, 75, 707-715.

Nock, M. K., Joiner, T. E., Gordon, K. H., Lloyd-Richardson, E., \& Prinstein, M. J. (2006). Non-suicidal self-injury among adolescents: Diagnostic correlates and relation to suicide attempts. Psychiatry Research, 144, 65-72.

Nock, M. K., Park, J. M., Finn, C. T., Deliberto, T. L., Dour, H. J., \& Banaji, M. R. (2010). Measuring the suicidal mind:
Cognition predicts suicidal behavior. Psychological Science, 21, 511-517.

Nock, M. K., \& Prinstein, M. J. (2004). A functional approach to the assessment of self-mutilative behavior. Journal of Consulting and Clinical Psychology, 72, 885-890.

Nock, M. K., \& Prinstein, M. J. (2005). Contextual features and behavioral functions of self-mutilation among adolescents. Journal of Abnormal Psychology, 114, 140-146.

Nock, M. K., Temper, R., \& Hollander, M. (2007). Psychological treatment of self-injury among adolescents. Journal of Clinical Psychology, 63, 1081-1089.

Prinstein, M. J., Nock, M. K., Simon, V., Aikins, J. W., Cheah, C. S. L., \& Spirito, A. (2008). Longitudinal trajectories and predictors of adolescent suicidal ideation and attempts following inpatient hospitalization. Journal of Consulting and Clinical Psychology, 76, 92-103.

Robins, L. N., \& Regier, D. A. (1991). Psychiatric Disorders in America: The Epidemiologic Catchment Area Study. New York: Free Press (McMillan).

Ross, S., \& Heath, N. (2002). A study of the frequency of selfmutilation in a community sample of adolescents. Journal of Youth and Adolescence, 31, 67-77.

Sim, L., \& Zeman, J. (2005). Emotion regulation factors as mediators between body dissatisfaction and bulimic symptoms in early adolescent girls. Journal of Early Adolescence, $25,478-496$.

Sobell, L. C., \& Sobell, M. B. (1992). Timeline followback: A technique for assessing self-reported alcohol consumption. In R. Z. Litten \& J. Allen (Eds.). Measuring alcohol consumption: Psychosocial and biological methods (pp. 41-72). Totowa, NJ: Humana Press.

Sobell, L. C., \& Sobell, M. B. (2003). Alcohol consumption measures. In J. P. Allen \& V. Wilson (Eds.). Assessing alcohol problems (pp. 75-99). (2nd ed.). Rockville, MD: National Institute on Alcohol Abuse and Alcoholism.

Spitzer, R. L., Kroenke, K., \& Williams, J. B. W. (1999). Validation and utility of a self-report version of Prime-MD: A PHQ primary care study. Journal of the American Medical Association, 282, 1787-1788.

Weinhardt, L. S., Carey, M. P., Maisto, S. A., Carey, K. B., Cohen, M. M., \& Wickramasinghe, S. M. (1998). Reliability of the timeline followback sexual behavior interview. Annals of Behavioral Medicine, 20, 25-30.

Whiteside, S. P., \& Lynam, D. R. (2001). The five factor model and impulsivity: Using a structural model of personality to understand impulsivity. Personality and Individual Differences, 30, 669-689.

Whitlock, J., Eckenrode, J., \& Silverman, D. (2006). Self-injurious behaviors in a college population. Pediatrics, 117, 1939-1948.

Wills, T. A., \& Shiffman, S. (1985). Coping and substance use: A conceptual framework. In S. Shiffman \& T.A. Wills (Eds.). Coping and substance use (pp. 451-458). Orlando, FL: Academic Press.

Yates, T. M., Tracy, A. J., \& Luthar, S. S. (2008). Nonsuicidal self-injury among "privileged" youths: Longitudinal and cross-sectional approaches to developmental process. Journal of Consulting and Clinical Psychology, 76, 52-62.

Zanarini, M. C., Vujanovic, A. A., Parachini, E. A., Boulanger, J. L., Frankenburg, F. R., \& Hennen, J. (2003). A screening measure for BPD: The McLean screening instrument for borderline personality disorder. Journal of Personality Disorders, 17, 568-573.

Received: November 9, 2010

ACCEPTED: April 17, 2011

Available online 12 June 2011 